\title{
New hope for patients with pure lower motor neuron syndromes
}

Motor neuron disease is still one of the most dramatic neurological conditions; it reflects a range of which one end encompasses patients with the bulbar form of amyotrophic lateral sclerosis who have dysarthria and dysphagia and have a dramatically shortened life expectancy. For patients with probable or definite motor neuron disease according to established criteria, the recent introduction of the antiglutamate drug riluzole gives a mean prolongation of life after diagnosis of some 3 months. ${ }^{1}$ The other end of the range of motor neuron disease is formed by patients with so called multifocal motor neuropathy. This is characterised by lower motor neuron dysfunction in the areas of selected motor nerves. Neurophysiological demonstration of conduction block over these nerves is pivotal for the diagnosis. ${ }^{2}$ Before the discovery of multifocal motor neuropathy as a separate and treatable condition, patients with the disease underwent the same fate as many other patients with motor neuron disease as they would have progressed into a state of severe disability. ${ }^{34}$ In the paper by Ellis et al (pp 15-19, this issue) the effect of the standard treatment of multifocal motor neuropathy-intravenous administration of human immunoglobulins - is analysed in patients with localised, pure lower motor neuron syndromes but no conduction block. Four of 10 patients seemed to benefit from treatment and three of these did so over prolonged periods of time. Most patients with multifocal motor neuropathy and conduction block experience their first symptoms in muscles of one forearm or hand, as did the four responders to immunoglobulins in this study. Four of the patients who did not respond had some evidence of respiratory dysfunction, which is uncommon for multifocal motor neuropathy. Increased muscle tendon reflexes and Babinski's sign always exclude the diagnosis, but the first may be difficult to interpret.

What is the value of this interesting study for the general neurologist? In our opinion there are three important points. Firstly, this study further broadens the range of motor neuron disease as patients may have multifocal motor neuropathy even without detectable conduction block. In multifocal motor neuropathy, effects of treatment on quality adjusted life-years have not yet been calculated. ${ }^{5}$ Even without these data it cannot reasonably be denied that expensive forms of treatment are justified in patients who fulfill strict clinical and neurophysiological criteria. ${ }^{2}$ However, if these neurophysiological criteria are applied too rigidly, patients may be kept from a possible beneficial treatment. So it is our task to develop better criteria for multifocal motor neuropathy. In this respect special emphasis should be given to the search for conduction block in proximal nerve segments, which may be more difficult to show in comparison with blocking over distal segments. Magnetic resonance imaging of the brachial plexus may also help to demonstrate foci of inflammation.

The second conclusion is that these criteria can only be developed in experienced hands. The incidence of motor neuron disease throughout the world is about 2.25/100 000 . $^{6}$ Ten per cent of these present with pure lower motor neuron syndromes: acquired progressive spinal muscular atrophy. In The Netherlands this adds up to some 35 and in the United Kingdom to some 125 new patients annually. Given the fact that the diagnosis of motor neuron disease is not correct in $5 \%$ of patients, ${ }^{6}$ in our opinion all patients with suspected lower motor neuron disease should be referred to highly specialised centres for further analysis.

The final conclusion is that long term treatment of multifocal motor neuropathy has again been shown to be effective.

One of the drawbacks of treatment of multifocal motor neuropathy with immunoglobulins are the high costs, which may mount up to some $£ 15000$ per patient-year. After diagnosis in a specialised centre, the green light for expensive long term treatment can be given. Long term evaluation of these rare patients, preferably in the form of multicentre studies of larger groups of patients may also help to elucidate pathogenic mechanisms. At present it has not yet been established whether inflammation is a primary disease mechanism. An alternative explanation may be that foci of inflammation of motor nerves occur as a reaction on primary degeneration of spinal cord motor nuclei as occurs in spinal muscular atrophy.

JOHN H J WOKKE

LEONARD H VAN DEN BERG

Department of Neurology, University Hospital Utrecht, PO Box 85500, 3508 GA Utrecht, The Netherlands

Correspondence to: John H J Wokke, Department of Neurology, University Hospital Utrecht, PO Box 85500, 3508 GA Utrecht, The Netherlands. email J.Wokke@neuro.azu.nl.

1 Bensimon G, Lacomblez L, Meininger V, on behalf of the ALS-Riluzole Study Group. A controlled trial of riluzole in amyotrophic lateral sclerosis. N Engl F Med 1994;330:585-91.

2 Van den Berg LH, Franssen H, Wokke JHJ. The long-term treatment of intravenous immunoglobulin treatment in multifocal motor neuropathy. Brain 1998;121:421-8.

3 Parry GJ, Clarke S. Multifocal acquired demyelinating neuropathy masquerading as motor neuron disease. Muscle Nerve 1988;11:103-7.

4 Lange DJ, Trojaborg W, Latov N, et al. Multifocal motor neuropathy with Lange DJ, Trojaborg W, Latov N, et al. Multifocal motor neuropathy with
conduction block: is it a distinct clinical entity? Neurology; 1992;42:497-505 5 Robine JM. Measuring the burden of disease. Lancet 1998;352:757-8.

6 Wokke JHJ. Diseases that masquerade as motor neuron disease. Lancet 1996;347:1347-8. 